\title{
Choose to move: The motivational impact of autonomy support on motor learning
}

\author{
Rebecca Lewthwaite $\cdot$ Suzete Chiviacowsky . \\ Ricardo Drews • Gabriele Wulf
}

Published online: 3 March 2015

(C) Psychonomic Society, Inc. 2015

\begin{abstract}
Numerous studies in the motor learning domain have demonstrated learning advantages of self-controlled practice relative to yoked conditions. In separate lines of evidence in the social-psychological literature, findings show that providing participants with task-relevant autonomy support or minor incidental choices can result in superior outcomes when compared with conditions that thwart autonomy or do not offer choice. We hypothesized that motor learning could be enhanced by providing learners with choices - even if those choices are unrelated to task performance. In Experiment 1, two groups of participants practiced a golf putting task. While one group (the choice group) was able to select the color of golf balls (white, yellow, or orange) to be used in each upcoming block of 10 trials, participants in the second group (the yoked group) were provided with the same colored golf balls their choice-group counterparts had chosen. The results of a 24-h delayed retention test indicated significantly greater putting accuracy for the choice compared with the yoked group. Experiment 2 went one step further by asking choice group participants for their preferences regarding two issues unrelated to the practice task (balancing on a stabilometer): (1) which of two subsequent tasks (coincident timing or hand dynamometry) they wanted to perform and (2) which of two prints of paintings by Renoir they thought the investigator should hang
\end{abstract}

R. Lewthwaite

Rancho Los Amigos National Rehabilitation Center, Downey, CA, USA

R. Lewthwaite

University of Southern California, Los Angeles, CA, USA

S. Chiviacowsky $\cdot$ R. Drews

Federal University of Pelotas, Pelotas, Brazil

G. Wulf $(\square)$

Department of Kinesiology and Nutrition Sciences, University of

Nevada, Las Vegas, 4505 Maryland Parkway, Las

Vegas, NV 89154-3034, USA

e-mail: gabriele.wulf@unlv.edu on the laboratory wall. Yoked group participants were simply informed about which task they would perform afterwards and of which painting the experimenter would put on the wall. Balance learning was significantly more effective in the choice group on a retention test. Thus, self-controlled practice conditions can influence motor learning without providing task-relevant information, content, or strategic learning advantages. Self-controlled effects in motor learning may be motivational in nature, attributable to satisfaction of fundamental autonomy needs.

Keywords Self-controlled practice $\cdot$ Fundamental psychological needs $\cdot$ Choice $\cdot$ Golf putting $\cdot$ Balance learning

The opportunity to exercise control over personal actions and factors that influence one's life appears to satisfy a basic psychological need (Deci \& Ryan, 2000, 2008; White, 1959) and may be considered a biological necessity (Langer \& Rodin, 1976; Leotti, Iyvengar, \& Ochsner, 2010; Leotti \& Delgado, 2011). Unique neural activations are associated with self- versus non-self-determined circumstances and a sense of agency or causality over actions and events (Lee \& Reeve, 2013; Leotti \& Delgado, 2014; Xue et al., 2014). Numerous studies have consistently shown that the learning of motor skills is enhanced when learners are given control over aspects of their practice conditions. Benefits of motor learner-controlled (often called self-controlled) practice have been found for a variety of putatively task-relevant conditions, including the delivery of feedback, augmented task information, assistive devices, movement demonstrations, and even the number of practice trials (for reviews, see Sanli, Patterson, Bray, \& Lee, 2013; Wulf, 2007). For example, when learners were given control over the use of assistive devices on balance tasks, learning was enhanced relative to the externally controlled use of the pole (yoked conditions) (Wulf, Clauss, Shea, \& 
Whitacre, 2001; Wulf \& Toole, 1999). In two studies (Chiviacowsky, Wulf, Lewthwaite, \& Campos, 2012; Hartman, 2007), learning to balance on a movable platform was facilitated by holding a balance pole in a horizontal position when the use of that pole was controlled by the learner. This finding is particularly interesting because, in a pilot study (Hartman, 2007), no advantages of using the poles were found for the learning of this task. This suggested that having choice over an assistive device can facilitate learning, even if that device in and of itself is relatively ineffective.

Many possible explanations have been proposed for the learning benefits of granting learners control over practice conditions. For example, it has been suggested that a more active involvement of the learner in the learning process promotes deeper processing of relevant information (Janelle, Barba, Frehlich, Tennant, \& Cauraugh, 1997) or that it encourages the use of self-regulation strategies with performance benefits (Baumeister, 1984; Kirschenbaum, 1987). Others have posed rationale more directly related to motivational influences, noting that giving the learner control over the practice conditions might be, in a general sense, more motivating (Chiviacowsky et al., 2012); or satisfy the learner's need for autonomy (Lewthwaite \& Wulf, 2012), which may enhance learning by increasing learners' perceived competence or self-efficacy (Chiviacowsky, Wulf, \& Lewthwaite, 2012; Tafarodi, Milne, \& Smith, 1999).

Interestingly, benefits of "choice" have been found even under conditions of chance in which illusions of control are generated (e.g., Langer, 1975; Xue et al., 2014). Furthermore, when choice involved incidental or trivial options - such as naming the characters in a computer game (Cordova \& Lepper, 1996) or choosing the names to be used in a short story (Tafarodi et al., 1999) - benefits for learning arithmetic tasks or reading comprehension, respectively, were observed.

In the present studies, we asked whether providing individuals with incidental choices in the context of motor skill acquisition could facilitate skill learning. In Experiment 1, participants practiced putting a golf ball. One group (choice) was provided the opportunity to choose the color of the golf balls they wanted to use before each 10-trial block, while (no-choice) participants were yoked to the ball color choices of their counterparts in the choice group. In Experiment 2, participants in one group (choices) were afforded two choices - both unrelated to the motor (balance) task to be learned - before the beginning of practice, while participants in a yoked group were given the respective information. In both studies, learning was measured by retention tests on the day following practice.

\section{Experiment 1}

In studies examining the influence of learner- or selfcontrolled relative to yoked practice conditions, the variables participants were able to control were typically related to task performance. For instance, participants were given the opportunity to request feedback about their performance (e.g., Janelle et al., 1997), or demonstrations of the goal task (e.g., Wulf, Raupach, \& Pfeiffer, 2005), choose their amount of practice (Post, Fairbrother, \& Barros, 2011), or select the level of task difficulty (Andrieux, Danna, \& Thon, 2012). Perhaps not surprisingly, interpretations of the learning advantages in learner-controlled groups have focused on learners' ability to tailor practice conditions to their needs (Chiviacowsky \& Wulf, 2002), or variations of this (information-processing) theme. In the present experiment, the choice learners were given was not related to task performance per se. Therefore, any learning benefits resulting from having, as opposed to not having, a choice would suggest that motivational factors are responsible for those effects.

\section{Method}

Participants Twenty-four university students (16 males, 8 females) with a mean age of 20.6 years (SD: 2.76) participated in this experiment. None of the participants had prior experience with the task, and all gave their informed consent before participating. The study was approved by the university's institutional review board.

Apparatus, task, and procedure The task consisted of participants putting a colored (white, orange, or yellow) regulation golf ball to a circular target with a radius of $10 \mathrm{~cm}$, placed on the floor at a distance of $4 \mathrm{~m}$ from the participant. Drawn around the target were concentric circles with radii of 20, 30, $40,50,60,70,80,90$, and $100 \mathrm{~cm}$, which served as zones of performance accuracy. A total of 100 points were awarded if the golf ball landed on the center bull's eye, and 90, 80, 70, 60, $50,40,30,20,10$, or 0 points were awarded if it landed in one of the other zones, or outside the circles.

Participants were quasi-randomly assigned to 2 different groups (choice and yoked), with equal numbers of females and males in each group. While participants in the choice group were informed that they could choose the color of the golf ball and change it (or not) after every 10 trials, according to their preferences, the participants of the yoked group were informed that the experimenter would determine the color of the golf ball to be used before each block of 10 trials. Participants of the yoked group were provided, without their knowledge, with the golf balls on the same color schedule chosen by their counterpart in the choice group. During the practice phase, all participants performed 60 trials. One day after this phase, participants performed a retention test, consisting of 10 trials, using only white balls.

Data analysis Accuracy scores, averaged across blocks of 10 trials, were analyzed with a 2 (group: choice versus yoked) $\times 6$ (blocks of 10 trials) analysis of variance (ANOVA), with 
repeated measures on the last factor for the practice phase. A one-way ANOVA was utilized for the retention test.

\section{Results}

Practice Both groups increased their putting accuracy across practice blocks (see Fig. 1, left), with the choice group tending to demonstrate greater accuracy than the yoked group on the last 4 of the 6 blocks. The main effect of trial was significant, $F(5,110)=8.53, p<.001, \eta_{p}{ }^{2}=.28$. The Group main effect approached significance, $F(1,22)=3.87, p=.065, \eta_{p}{ }^{2}=.15$, while the interaction of group and trial was not significant, $F(5,110)=1.76, p=.13$.

Retention On the retention test, during which white golf balls were used, the choice group showed significantly higher putting accuracy $(36.8)$ than the yoked group $(26.4), F(1,22)=$ $7.31, p<.05, \eta_{p}{ }^{2}=.25$ (see Fig. 1, right).

\section{Discussion}

Giving learners a choice about the golf ball color (on six occasions throughout the practice phase) enhanced the learning of the golf putting task for choice-group participants relative to a group of learners who were not provided a choice. While the benefits of learner-controlled practice for motor learning have been documented for a wide range of tasks and various factors controlled by learners (see Sanli et al., 2013, for a review), the present finding shows that even a choice that is incidental to the task can enhance motor skill learning. This result cannot be explained by practice conditions being optimized for individual learners' information-processing needs. Rather, the satisfaction of a more fundamental psychological or biological need for autonomy (e.g., Deci \& Ryan, 2000; Leotti \& Delgado, 2011) may explain the learning advantages of learnercontrolled practice conditions.

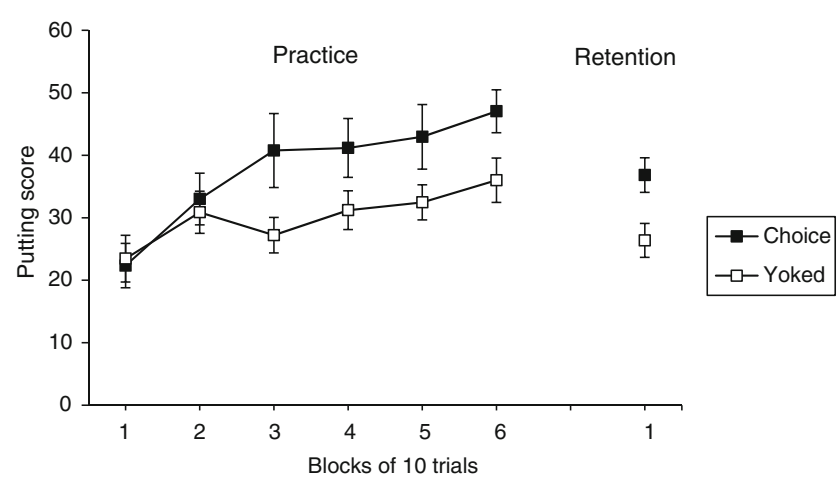

Fig. 1 Putting accuracy (higher scores indicate greater accuracy) of the choice and yoked groups during practice and retention (Experiment 1). Error bars standard errors

\section{Experiment 2}

Given the potential theoretical importance of the finding in Experiment 1, we wanted to replicate it with another task and different type of choice. Despite the fact that the golf ball color would not be expected to influence putting accuracy, we cannot exclude the possibility that participants had, or developed, expectations about a particular ball color "working" better than others, which in turn may have affected putting accuracy (Damisch, Stoberock, \& Mussweiler, 2010). Therefore, we went one step further in Experiment 2 and examined whether choices more distinctly unrelated to the task would have an impact on task learning.

Method

Participants Thirty university students (18 males, 12 females) with a mean age of 21.1 years (SD: 2.02) participated in this experiment. Participants were not aware of the purpose of the study and had no prior experience with the experimental task. All participants gave their informed consent and the study was approved by the university's institutional review board.

Apparatus and task The task required participants to balance on a movable balance platform or stabilometer. The $130 \times$ $140 \mathrm{~cm}$ wooden platform had a maximum deviation of $18^{\circ}$ to either side. The participant's task was try to keep the platform as close to the horizontal position as possible during each 30 -s trial. Time in balance (i.e., platform position within $\pm 5^{\circ}$ from horizontal) was measured by a millisecond timer.

Procedure Participants were quasi-randomly (based on gender) assigned to either the choice or the no-choice group, with 6 women in each group. They were informed that the aim of the task was to keep the platform as close to horizontal as possible and that they would be given feedback about their time in balance after each practice trial.

Before the beginning of the practice phase, participants in the choice group were presented with two choices unrelated to the primary motor task: (1) which of two tasks [a coincidenttiming task (Bassin timer) or a hand dynamometry task] they preferred as a second task to be performed on the next day, after finishing their trials on the primary task; and (2) which of two prints of paintings by the artist Renoir they thought the investigator should hang on the laboratory wall. Participants in the no-choice group were told that: (1) there were two tasks which could be performed by the participants on the next day, after finishing their trials on the primary task (i.e., coincidenttiming task or hand dynamometry task), but that the experimenter had already chosen the task they would perform; and (2) the experimenter had pondered which of two prints of paintings by Renoir to hang on the laboratory wall, but that the choice had already been made. The specific second task 
and painting chosen were pointed out to them within the laboratory.

Immediately after that designation (choice) or indication (no-choice), all participants performed 10 trials of the stabilometer task, with a duration of $30 \mathrm{~s}$ each, and 90-s rest intervals between trials. Feedback about time in balance (in s) was provided after each practice trial. A retention test consisting of 530 -s trials with 90 -s breaks between trials, without feedback, was conducted $24 \mathrm{~h}$ later to assess learning effects as a function of practice conditions. To close the experimental session and provide participants with the expected practice of another task, all participants completed the secondary task. However, those results were not considered for analysis as they occurred following all trials on the primary task and did not fit the experimental purpose.

Data analysis Time in balance for the practice phase was analyzed in a 2 (groups) $\times 10$ (trials) analysis of variance (ANOVA) with repeated measures on the last factor, while the retention data were analyzed in a 2 (groups) $\times 5$ (trials) repeated-measures ANOVA.

\section{Results}

Practice Time in balance increased in both groups across practice trials (see Fig. 2, left). The main effect of trial was significant, $F(9,243)=38.62, p<.001, \eta_{p}{ }^{2}=.59$. The choice group tended to increase their times at a somewhat faster pace than the yoked group. However, the main effect of group, $F(1$, $27)=2.13, p>.05$, and the interaction of group and trial were not significant, $F(9,243)<1$.

Retention On the retention test 1 day later, the choice group demonstrated significantly longer times in balance than the yoked group, $F(1,27)=7.93, p<.01, \eta_{p}{ }^{2}=.23$ (see Fig. 2 , right). Also, both groups continued to increase their time in balance, $F(4,108)=6.38, p<.001, \eta_{p}{ }^{2}=.19$. The interaction of group and trial was not significant, $F(4,108)<1$.

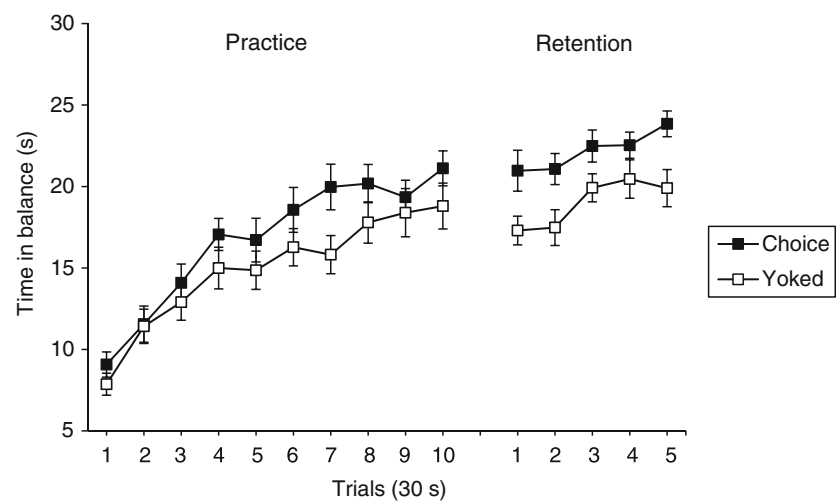

Fig. 2 Balance performance (time in balance) of the choice and yoked groups during practice and retention (Experiment 2). Error bars standard errors
Discussion

Incidental choices, provided to participants before practicing a novel balance task, enhanced the learning of that skill. In previous studies, giving participants choices that were unrelated to task performance - but still linked to the task in small ways (Cordova \& Lepper, 1996; Tafarodi et al., 1999; Experiment 1 here) - enhanced performance relative to not providing choices. The present results show that even small and incidental choices arguably unrelated to the task can facilitate learning. This finding provides further evidence for the notion that satisfying learners' need for autonomy is beneficial in the context of learning.

\section{General discussion}

The desire to experience a sense of agency or to be in control of one's life circumstances has been recognized as a fundamental need (e.g., Deci \& Ryan, 2000, 2008; Leotti et al., 2010; Leotti \& Delgado, 2011), and its fulfillment has been linked to increases in engagement (Leotti \& Delgado, 2014), performance (Cordova \& Lepper, 1996; Tafarodi et al., 1999), and quality of life (Langer \& Rodin, 1976). Similarly, in the motor learning domain, numerous studies over the past two decades have demonstrated that motor skill learning is enhanced when learners are given choices about aspects of the task or conditions of practice (i.e., self-controlled practice). A recent meta-analysis of 23 such studies indicates an effect size of 0.63 for the impact of self-controlled relative to yoked conditions of practice on learning as reflected in delayed retention test performance (McKay, Carter, \& Ste-Marie, 2014). Yet, only recently have those learning effects been discussed as consistent with functioning to support learners' needs for autonomy (Lewthwaite \& Wulf, 2012; Sanli et al., 2013). The present results appear to be among the first to provide direct evidence that giving learners choice per se can affect motor learning without providing content (e.g., demonstrations of the goal movement) or strategic learning advantages (e.g., use of assistive devices to help explore a new movement strategy). Moreover, our findings show that even minor choices that are ostensibly unrelated to the motor task - can affect learning. The present findings also extend previous findings in which task performance was facilitated by giving participants (task-related) choices (Cordova \& Lepper, 1996; Tafarodi et al., 1999). As is common practice in the motor learning research, we used delayed retention tests as a measure of learning, in which all groups perform under identical conditions (i.e., white golf balls in Experiment 1; no choices in both experiments). A 1-day delay (or more) is typically used in motor learning research to allow for the dissipation of possible temporary and spurious effects of the independent variables on performance (Schmidt \& Lee, 2011). Further, this 
delay may provide an opportunity for memory consolidation through sleep and wakefulness processes and other influences that strengthen motor memory (Albouy, King, Maquet, \& Doyon, 2013; Robertson, 2009). Thus, the present findings indicate that providing choices during, or before, practice can have lasting effects on the retention of motor skills.

Through which mechanisms does the capability to make choices operate to promote learning? One effect of autonomysupportive conditions might be that they convey a general sense of respect for participants' agency or capabilities that, in turn, increases learners' generalized confidence and thereby task-specific self-efficacy (Hooyman, Wulf, \& Lewthwaite, 2014; Wulf, Chiviacowsky, \& Cardozo, 2014). Instructions that suggested some freedom in how participants approached a novel cricket bowling task led to better learning and higher self-efficacy for accurate bowling than controlling instructions impinging upon autonomy in task execution (Hooyman et al., 2014). Alternatively, letting participants make even apparently insignificant and inconsequential choices such as asking for their opinion, as in the second experiment here, may lessen needs for even minor defensive, resistive, or anxious activation, laying the groundwork for less impeded task-focused activity.

Furthermore, exercising control and the prospective opportunity to do so appears to be inherently rewarding (Fujiwara et al., 2013; Leotti \& Delgado, 2011, 2014). Choice, or the anticipation of the opportunity for choice, is associated with increased activity of brain regions directly involved in reward processing (Fujiwara et al., 2013; Leotti \& Delgado, 2011, 2014). Greater interest in learning a task (Chiviacowsky et al., 2012) and emotional engagement (Leotti \& Delgado, 2014; Reeve \& Tseng, 2011) may be indicative of this reward function. Rewards can trigger dopaminergic responses (Hosp \& Luft, 2013; Schultz, 2013) and support memory consolidation processes (Abe et al., 2011; Adcock, Thangavel, Whitfield-Gabrieli, Knutson, \& Gabrieli, 2006; Sugawara, Tanaka, Okazaki, Watanabe, \& Sadato, 2012).

The present studies suggest that the experience of choice, without regard for the apparently inconsequential or incidental contents of the choice, is sufficient to trigger enhancement of motor learning. The finding that choices as incidental to the to-be-learned activity as golf ball color, subsequent tasks of very different motoric demands (e.g., anticipatory timing via a hand-held button or grip force modulation) to be performed a day later, and artwork selection affect motor learning is consistent with literature in other fields of human endeavor or experience. The majority of research in the motor learning self-controlled practice literature, however, has assumed that there is particular informational value governing the influence of learner-controlled conditions. Indeed, most conditions, such as the opportunity to select trials for feedback, when to view models demonstrating the task, or when to use assistive devices have had arguably beneficial roles in motor performance. Few studies have examined self-control of conditions that either have little or no apparent relationship to the task at hand. Exceptions in this regard include several studies by Post and colleagues (Post et al., 2011; Post, Fairbrother, Barros, \& Kulpa, 2014) in which self-controlled opportunities for the number of practice attempts varied considerably between participants but resulted in better transfer or retention test performance for learner-controlled than yoked conditions. The number of practice trials is typically accorded a preeminent role among factors determining the extent of motor learning (e.g., Schmidt \& Lee, 2011), yet fewer trials, if selfchosen, resulted in more effective learning than more trials imposed upon learners (Post et al., 2011, 2014).

That six occasions of ball color choice (Experiment 1) and two opportunities for expressing preferences (Experiment 2) were sufficient to affect motor learning suggests that the mechanism underlying previously found advantages of selfcontrolled and task-relevant conditions of practice (see McKay et al., 2014; Wulf, 2007) may be more elemental and motivational in nature. That is, they may not pertain to creating a better fit between the learner and unfolding task-related processing, strategic, content, or other performance needs. The common denominator within the set of motor learning studies may simply be the intrinsically rewarding opportunity to exercise or anticipate control, or choice, of any kind (presumably at least somewhat meaningful and respectful in nature) - although information-processing differences may well follow motivational triggers (Graham \& Golan, 1991). Of course, the types of self-controlled aspects of practice examined in the motor learning literature to date would provide learners a sense of agency as well, and perhaps more in terms of task-specific benefits. When substantial choices do not seem indicated, however, small choices may suffice to potentiate motor learning (see also Wulf \& Adams, 2014).

\section{References}

Abe, M., Schambra, H., Wassermann, E. M., Luckenbaugh, D., Schweighofer, N., \& Cohen, L. G. (2011). Reward improves longterm retention of a motor memory through induction of offline memory gains. Current Biology. doi:10.1016/j.cub.2011.02.030

Adcock, R. A., Thangavel, A., Whitfield-Gabrieli, S., Knutson, B., \& Gabrieli, J. D. E. (2006). Reward-motivated learning: Mesolimbic activation precedes memory formation. Neuron, 50, 507-517. doi: 10.1016/j.neuron.2006.03.036

Albouy, G., King, B. R., Maquet, P., \& Doyon, J. (2013). Hippocampus and striatum: Dynamics and interaction during acquisition and sleep-related motor sequence memory consolidation. Hippocampus, 23, 985-1004.

Andrieux, M., Danna, J., \& Thon, B. (2012). Self-control of task difficulty during training enhances motor learning of a complex coincidence-anticipation task. Research Quarterly for Exercise and Sport, 83, 27-35. 
Baumeister, R. F. (1984). Choking under pressure: Self-consciousness and paradoxical effects of incentives on skillful performance. Journal of Personality and Social Psychology, 46, 610-620.

Chiviacowsky, S., \& Wulf, G. (2002). Self-controlled feedback: Does it enhance learning because performers get feedback when they need it? Research Quarterly for Exercise and Sport, 73, 408-415.

Chiviacowsky, S., Wulf, G., Lewthwaite, R., \& Campos, T. (2012). Motor learning benefits of self-controlled practice in persons with Parkinson's disease. Gait \& Posture, 35, 601-605.

Chiviacowsky, S., Wulf, G., \& Lewthwaite, R. (2012). Self-controlled learning: The importance of protecting perceptions of competence. Frontiers in Psychology, 3(Article 458). doi:10.3389/fpsyg.2012. 00458

Cordova, D. I., \& Lepper, M. R. (1996). Intrinsic motivation and the process of learning: Beneficial effects of contextualization, personalization, and choice. Journal of Educational Psychology, 88, 715730 .

Damisch, L., Stoberock, B., \& Mussweiler, T. (2010). Keep your fingers crossed! How superstition improves performance. Psychological Science, 21, 1014-1020.

Deci, E. L., \& Ryan, R. M. (2000). The "what" and "why" of goal pursuits: Human needs and the self-determination of behavior. Psychological Inquiry, 11, 227-268.

Deci, E. L., \& Ryan, R. M. (2008). Self-determination theory: A macrotheory of human motivation, development, and health. Canadian Psychology, 49, 182-185.

Fujiwara, J., Usui, N., Park, S. Q., Williams, T., Iijima, T., Taira, M., ... Tobler, P. N. (2013). Value of freedom to choose encoded by the human brain. Journal of Neurophysiology, 110, 1915-1929.

Graham, S., \& Golan, S. (1991). Motivational influences on cognition: Task involvement, ego involvement, and depth of information processing. Journal of Educational Psychology, 83, 187-194.

Hartman, J. M. (2007). Self-controlled use of a perceived physical assistance device during a balancing task. Perceptual and Motor Skills, 104, 1005-1016.

Hooyman, A., Wulf, G., \& Lewthwaite, R. (2014). Impacts of autonomysupportive versus controlling instructional language on motor learning. Human Movement Science, 36, 190-198.

Hosp, J. A., \& Luft, A. R. (2013). Dopaminergic meso-cortical projections to M1: Role in motor learning and motor cortex plasticity. Frontiers in Neurology, 4, 145. doi:10.3389/fneur.2013.00145

Janelle, C. M., Barba, D. A., Frehlich, S. G., Tennant, L. K., \& Cauraugh, J. H. (1997). Maximizing performance effectiveness through videotape replay and a self-controlled learning environment. Research Quarterly for Exercise and Sport, 68, 269-279.

Kirschenbaum, D. S. (1987). Self-regulation of sport performance. Medicine \& Science in Sports \& Exercise, 19(5, Supplement), S106-S113.

Langer, E. J. (1975). The illusion of control. Journal of Personality and Social Psychology, 32, 311-328.

Langer, E. J., \& Rodin, J. (1976). The effects of choice and enhanced personal responsibility for the aged: A field experiment in an institutional setting. Journal of Personality and Social Psychology, 34, 191-198.

Lee, W., \& Reeve, J. (2013). Self-determined, but not non-self-determined, motivation predicts activations in the anterior insular cortex: An fMRI study of personal agency. Social Cognitive and Affective Neuroscience, 8, 538-545.

Leotti, L. A., \& Delgado, M. R. (2011). The inherent reward of choice. Psychological Science, 22, 1310-1318.

Leotti, L. A., \& Delgado, M. R. (2014). The value of exercising control over monetary gains and losses. Psychological Science, 25, 596604.
Leotti, L. A., Iyvengar, S. S., \& Ochsner, K. N. (2010). Born to choose: The origins and value of the need for control. Trends in Cognitive Science, 14, 457-463.

Lewthwaite, R., \& Wulf, G. (2012). Motor learning through a motivational lens. In N. J. Hodges \& A. M. Williams (Eds.), Skill acquisition in sport: Research, theory \& practice (2nd ed., pp. 173-191). London: Routledge.

McKay, B., Carter, M. J., \& Ste-Marie, D. (2014, June). Self-controlled learning: A meta-analysis. Journal of Sport \& Exercise Psychology, 36(Suppl), S43.

Post, P. G., Fairbrother, J. T., Barros, J. A. C., \& Kulpa, D. A. (2014). Self-controlled practice within a fixed time period facilitates the learning of a basketball set shot. Journal of Motor Learning and Development, 2, 9-15.

Post, P. G., Fairbrother, J. T., \& Barros, J. A. C. (2011). Self-controlled amount of practice benefits learning of a motor skill. Research Quarterly for Exercise and Sport, 82, 474-481.

Reeve, J., \& Tseng, C. M. (2011). Cortisol reactivity to a teacher's motivating style: The biology of being controlled versus supporting autonomy. Motivation and Emotion, 35, 63-74.

Robertson, E. M. (2009). From creation to consolidation: A novel framework for memory processing. PLoS Biology, 7(1), e1000019. doi: 10.1371/journal.pbio.1000019

Sanli, E. A., Patterson, J. T., Bray, S. R., \& Lee, T. D. (2013). Understanding self-controlled motor learning protocols through self-determination theory. Frontiers in Psychology, 3(Article 611). doi:10.3389/fpsyg.2012.00611

Schmidt, R. A., \& Lee, T. D. (2011). Motor control and learning (5th ed.). Champaign: Human Kinetics.

Schultz, W. (2013). Updating dopamine reward signals. Current Opinion in Neurobiology, 23, 229-238.

Sugawara, S. K., Tanaka, S., Okazaki, S., Watanabe, K., \& Sadato, N. (2012). Social rewards enhance offline improvements in motor skill. PLoS ONE, 7(11), e48174. doi:10.1371/journal/pone.0048174

Tafarodi, R. W., Milne, A. B., \& Smith, A. J. (1999). The confidence of choice: Evidence for an augmentation effect on self-perceived performance. Personality and Social Psychology Bulletin, 25, 14051416.

White, R. W. (1959). Motivation reconsidered: The concept of competence. Psychological Review, 66, 297-333.

Wulf, G. (2007). Self-controlled practice enhances motor learning: Implications for physiotherapy. Physiotherapy, 93, 96-101.

Wulf, G., \& Adams, N. (2014). Small choices can enhance balance learning. Human Movement Science, 38, 235-240.

Wulf, G., Chiviacowsky, S., \& Cardozo, P. (2014). Additive benefits of autonomy support and enhanced expectancies for motor learning. Human Movement Science, 37, 12-20.

Wulf, G., Clauss, A., Shea, C. H., \& Whitacre, C. (2001). Benefits of selfcontrol in dyad practice. Research Quarterly for Exercise and Sport, 72, 299-303.

Wulf, G., Raupach, M., \& Pfeiffer, F. (2005). Self-controlled observational practice enhances learning. Research Quarterly for Exercise and Sport, 76, 107-111.

Wulf, G., \& Toole, T. (1999). Physical assistance devices in complex motor skill learning: Benefits of a self-controlled practice schedule. Research Quarterly for Exercise and Sport, 70, 265-272.

Xue, G., He, Q., Lu, Z.-L., Levin, I. P., Dong, Q., \& Bechara, A. (2014). Agency modulates the lateral and medial prefrontal cortex responses in belief-based decision making. PLOS ONE, 8(6), e65274. doi:10. 1371/journal.pone.0065274 